\title{
Cuarentena sola o combinada con otras medidas de salud pública para controlar COVID-19: revisión rápida
}

\author{
Quarantine alone or in combination with other public health measures to control COVID-19: a rapid review
}

\section{Comentado de:}

Nussbaumer-Streit B, et al. Cochrane Database of Systematic Reviews 2020, Issue 4. Art. No.: CD013574. PMID: $32267544^{1}$

\section{Antecedentes}

La enfermedad por coronavirus 2019 (COVID-19) es una en fermedad emergente que ha sido clasificada como pandemia por la Organización Mundial de la Salud (OMS) en marzo de 2020.

\section{Objetivos}

Se realizó una revisión rápida para evaluar los efectos de la cuarentena (sola o combinada con otras medidas) de individuos que tuvieron contacto con casos confirmados de COVID-19, que viajaron desde países con un brote declarado o que viven en regiones con alta transmisión de la enfermedad.

\section{Métodos de búsqueda}

Un especialista en información buscó en PubMed, Ovid MEDLINE, OMS Global Index Medicus, Embase y CINAHL el 12 de febrero de 2020 y actualizó la búsqueda el 12 de marzo de 2020. La OMS proporcionó registros de búsquedas diarias en bases de datos chinas hasta el 16 de marzo de 2020.

\section{Criterios de selección}

Se incluyeron estudios de cohortes, estudios caso-control, series de casos, series de tiempo, series de tiempo interrumpidas y estudios de modelos matemáticos que evaluaron el efecto de cualquier tipo de cuarentena para controlar COVID-19. También se incluyeron estudios sobre el SARS (síndrome respiratorio agudo severo) y el MERS (síndrome respiratorio del Medio Oriente) como evidencia indirecta del brote actual de coronavirus (denominado SARS-CoV-2).

\section{Recolección y análisis de datos}

Dos revisores seleccionaron de forma independiente el $30 \%$ de los registros y un solo revisor examinó el $70 \%$ restante. Dos revisores seleccionaron todas las publicaciones de texto completo potencialmente relevantes de forma independiente. Un autor de la revisión extrajo los datos y evaluó la calidad de la evidencia con GRADE y un segundo autor de la revisión verificó la evaluación. Se calificó la confianza en la evidencia de los cuatro resultados primarios: incidencia, transmisión posterior, mortalidad y uso de recursos.

\section{Resultados principales}

Se incluyeron 29 estudios: diez estudios de modelado sobre COVID-19, cuatro estudios observacionales (178.122 personas involucradas en brotes de SARS y MERS) y 15 estudios de modelado sobre SARS y MERS. Debido a los diversos métodos de medición y análisis de los resultados de interés, no se pudo realizar un meta-análisis, y se realizó una síntesis narrativa. Debido al tipo de evidencia encontrada para esta revisión, GRADE califica la confianza en la evidencia como baja a muy baja.

Los estudios de modelado informaron, de manera consistente, un beneficio de las medidas de cuarentena simuladas, por ejemplo, la cuarentena de personas expuestas a casos confirmados o sospechosos evitó $44 \%$ a $81 \%$ de casos incidentes y $31 \%$ a $63 \%$ de muertes en comparación con ninguna medida, basados en diferentes escenarios (casos incidentes: 4 estudios de modelado sobre COVID-19, SARS; mortalidad: 2 estudios de modelado sobre COVID-19, SARS, evidencia de baja confianza) Evidencia de muy baja confianza sugiere que cuanto más temprano se implementen las medidas de cuarentena, mayor será el ahorro de costos (2 estudios de modelado sobre SARS). Evidencia de muy baja confianza indicó que el efecto de la cuarentena de los viajeros de un país con un brote declarado en la reducción de la incidencia y las muertes fue pequeño (2 estudios de modelado sobre el SARS). Cuando los modelos combinaron la cuarentena con otras medidas de prevención y control, incluido el cierre de escuelas, restricciones para viajar y distanciamiento social, se observó un mayor efecto en la reducción de nuevos casos, transmisiones y muertes que las medidas individuales por sí solas (casos incidentes: 4 estudios de modelado en COVID-19; transmisión prospectiva: 2 estudios de modelado en COVID-19; mortalidad: 2 estudios de modelado sobre COVID-19; evidencia de baja confianza). Los estudios sobre SARS y MERS fueron consistentes con los hallazgos de los estudios sobre COVID-19.

\section{Conclusiones de los autores}

La actual evidencia para COVID-19 se limita a los estudios de modelado, que hacen suposiciones de parámetros basadas en el fragmentado conocimiento disponible. Los resultados indican, consistentemente, que la cuarentena es importante para reducir la incidencia y la mortalidad durante la pandemia de COVID-19. La implementación temprana de la cuarentena y la combinación de ésta con otras medidas de salud pública es importante para garantizar su efectividad. Para mantener el mejor equilibrio posible en las medidas, los encargados de tomar decisiones deben monitorear constantemente la situación del brote y el impacto de las estrategias implementadas. Evaluar muestras representativas, en diferentes entornos, podría ayudar a dimensionar la verdadera prevalencia de la infección y reduciría la incertidumbre de los supuestos empleados en el modelado.

Fuente de financiamiento: Esta revisión fue encargada por la OMS y apoyada por Danube - University - Krems.

\section{Comentario}

Hasta la fecha, esta es la primera síntesis de evidencia sistemática rápida sobre la efectividad de las medidas de cuarentena para COVID-19 ${ }^{1}$. Todos los estudios de modelos encontraron, consistentemente, que las medidas de cuarentena simuladas podrían reducir el número de personas con COVID-19 y las muertes relacionadas hasta el $80 \%$ y $60 \%$, respectivamente. Una asunción central de los modelos es el número de reproducción efectiva, o R0, que es la cifra que define el número de casos secundarios generados por un caso primario. Para que se produzca una epidemia, el valor R0 debe ser mayor que 1. Cuando este se sitúa por debajo de 1, indica que existe control de la situación. Hay enfermedades muy contagiosas, como el 
sarampión, cuyo $\mathrm{R} 0=15$. La gripe tiene un $\mathrm{R}_{0}=1,3$ mientras que el de COVID-19 se estima entre 2,8 y $5,5^{2-4}$, de modo que una persona infectada, tenga síntomas o no, contagia en promedio entre 3 y 6 personas.

El conocido estudio del Colegio Imperial del Reino Unido mostró que una combinación de aislamiento de casos y cuarentena voluntaria durante tres meses podría prevenir el $31 \%$ de las muertes en comparación con una epidemia sin ninguna medida de control. Agregar el distanciamiento social de las personas de 70 años o más durante cuatro meses aumentaría la proporción de muertes evitadas al $49 \%\left(R_{0}=2,4\right.$ asumido). Consideraron que la combinación de aislamiento de casos, cuarentena doméstica, distanciamiento social de toda la población y cierres de escuelas y universidades es la combinación más efectiva que podría reducir el número de reproducción a un valor cercano a $1^{5}$.

Aunque las medidas de prevención y control más exhaustivas y estrictas son más efectivas para contener el COVID-19, en algún momento el efecto incremental de agregar otra medida restrictiva es solo mínimo, y éste debe contrastarse con los efectos negativos colaterales, como las consecuencias sociales y económicas para las comunidades que han estado sujetas a las medidas por períodos prolongados, lo que también podrían conducir a un aumento de la carga sobre la salud en general.

Los modelos han mostrado que la efectividad de poner en cuarentena a los individuos durante los brotes de enfermedades con infecciosidad presintomática y un $\mathrm{R}_{0}$ mayor a 2,5 es limitada por sí sola y puede requerir medidas de control adicionales ${ }^{2}$. Sin embargo, en una situación con infecciosidad presintomática o incluso asintomática, es difícil identificar y aislar a todos los casos y poner los contactos de los casos en cuarentena lo suficientemente temprano como para reducir notablemente la transmisión.

No podemos estar completamente seguros acerca de la evidencia encontrada por varias razones. Aunque una revisión rápida toma algunos atajos en pro de la oportunidad de la evidencia, la metodología empleada es lo suficientemente buena como para confiar en la calidad del proceso. Los estudios sobre COVID-19 basaron sus modelos en datos limitados e hicieron diferentes asunciones sobre el virus (por ejemplo, sobre la rapidez de su propagación, o sobre la adherencia a la cuarentena). Los otros estudios investigaron el SARS y el MERS, por lo que no podríamos asumir que los resultados serían los mismos para COVID-19 y la confianza en la evidencia se debilita por ser indirecta.

Sin embargo, la estimación de mayor costo-efectividad a más rápido inicio de la cuarentena, y de mayor efectividad al combinarla con otras medidas parecen darle un soporte adicional a los resultados favorables encontrados.

De todas maneras, una lección aprendida dolorosamente con la pandemia de COVID-19 es que, para mantener el mejor equilibrio posible en las medidas que se adopten, los decisores deben monitorear constantemente la evolución del brote y el impacto de las medidas implementadas. La versatilidad para hacer ajustes a corto plazo, comprendiendo el dinamismo de la situación, es clave para controlarla exitosamente. De esta implicancia práctica también se derivan recomendaciones para la investigación, como la estimación de la prevalencia, la tasa de letalidad, el impacto en los sistemas de salud y en la economía de los países y las personas. Las pruebas en muestras representativas y en diferentes ámbitos podrían ayudar también a reducir en gran medida la incertidumbre de las simulaciones de los modelos COVID-19. Diferentes países en todo el mundo han estado implementando combinaciones de medidas de prevención y de control, a diferente intensidad y velocidad. Serán muy útiles los estudios cuasi-experimentales, como las series de tiempo interrumpidas, que evalúen la efectividad de la cuarentena sola o en combinación con otras medidas de salud pública para controlar esta enfermedad.

\section{Conclusiones del comentador}

A pesar de la evidencia limitada, todos los estudios encontraron que la cuarentena es importante para reducir la cantidad de personas infectadas y la cantidad de muertes, siendo más efectiva y menos costosa cuando esta estrategia se inicia tempranamente. La combinación de la cuarentena con otras medidas de prevención y control tuvo un efecto mayor que la cuarentena sola.

Los modelos no han considerado aun un jugador que cada vez parece más cercano, como la aparición de una vacuna, que modificará definitivamente el tablero. Hasta que la pandemia sea definitivamente controlada, los decisores de salud pública deberán hacer un seguimiento estrecho del brote y del impacto de las medidas implementadas, e informar adecuadamente a sus poblaciones a cargo.

Agustín Ciapponi [ Centro Cochrane Argentina, Instituto de Efectividad Clínica y Sanitaria; Servicio de Medicina Familiar y Comunitaria, Hospital Italiano de Buenos Aires. aciapponi@iecs.org.ar ]

Ciapponi A Cuarentena sola o combinada con otras medidas de salud pública para controlar COVID-19: revisión rápida. Evid Actual Pract Ambul. 2020;23(1):e002054. Comentado de: Nussbaumer-Streit B, et al. Quarantine alone or in combination with other public health measures to control COVID19: a rapid review. Cochrane Database of Systematic Reviews 2020, Issue 4. Art. No.: CD013574. PMID: 32267544

\section{Referencias}

1. Nussbaumer-Streit B, Mayr V, Dobrescu Al, et al. Quarantine alone or in combination with other public health measures to control COVID-19: a rapid review. Cochrane Database of Systematic Reviews. 2020;2020(4). Available from: 10.1002/14651858.cd013574;https://dx.doi.org/10.1002/ 14651858.cd013574.

2. Read JM, Bridgen JR, Cummings DA, et al. Novel coronavirus 2019-nCoV: early estimation of epidemiological parameters and epidemic predictions. medRxiv. 2020;Available from: 10.1101/2020.01.23.20018549.

3. Zhou T, Liu Q, Yang Z, et al. Preliminary prediction of the basic reproduction number of the Wuhan novel coronavirus 2019-nCoV. J Evid Based Med. 2020;13(1):3-7. Available from: 10.1111/jebm.12376. 
4. Zhao S, Lin Q, Ran J, et al. Preliminary estimation of the basic reproduction number of novel coronavirus (2019-nCoV) in China, from 2019 to 2020 A data-driven analysis in the early phase of the outbreak. Int J Infect Dis. 2020;92:214-217. Available from: 10.1016/j.ijid.2020.01.050.

5. Ferguson NM, Laydon D, Nedjati-Gilani G, et al.. Impact of non-pharmaceutical interventions (NPIs) to reduce COVID19 mortality and healthcare demand; 2020. Available from: https://www.imperial.ac.uk/media/imperial-college/medicine/sph/ide/gida-fellowships/Imperial-College-COVID19NPI-modelling-16-03-2020.pdf [Last access: 2020-03-09]. 